\title{
Screening and Analysis of Multiclass Veterinary Drug Residues in Animal Source Foods using UPLC-Q-Exactive Orbitrap/MS
}

\author{
Wentao Zhao ${ }^{1} \cdot$ Rui Jiang ${ }^{1} \cdot$ Wenping Guo ${ }^{1} \cdot$ Chao Guo $^{1} \cdot$ Shilei $\mathrm{Li}^{1} \cdot$ Juanqiang Wang ${ }^{1} \cdot$ Shouwei Wang ${ }^{1}$. \\ Yingying $\mathrm{Li}^{1}{ }^{10}$
}

Received: 30 October 2020 / Accepted: 17 May 2021 / Published online: 7 June 2021

(c) The Author(s) 2021

\begin{abstract}
A rapid, simple, and sensitive method of detecting veterinary drug residues in animal food sources, including poultry and pork, was developed and validated. The method was optimized for over 155 veterinary drugs of 21 different classes. Sample pretreatment included a simple solid-liquid extraction step with $0.2 \%$ formic acid-acetonitrile-water and a purification step with a PRiME HLB (hydrophile-lipophile balance) solid-phase extraction cartridge. Data were collected using ultra-highperformance liquid chromatography coupled to Quadrupole-Exactive Orbitrap mass spectrometry. The limits of detection of 155 veterinary drugs ranged from $0.1 \mu \mathrm{g} / \mathrm{kg}$ to $10 \mu \mathrm{g} / \mathrm{kg}$. The recovery rates were between 79.2 and $118.5 \%$ in all matrices studied, with relative standard deviation values less than $15 \%(n=6)$. The evaluated method allows the reliable screening, quantification, and identification of 155 veterinary drug residues in animal source food and has been successfully applied in authentic samples.
\end{abstract}

Keywords UPLC-Q-Exactive Orbitrap/MS · Veterinary drug residues · Animal source foods · Qualitative screening · Quantitative determination

Meat is an indispensable part of the human diet (Laranjo et al. 2017). During breeding, animals are supplemented with veterinary drugs to treat and prevent diseases (Kpodékon et al. 2015; Knap 2020). However, excessive use and misuse of veterinary drugs can lead to human health risks and environmental pollution (Liu et al. 2019; Trishna et al. 2018). In recent years, food contamination by veterinary drug residue has become public health concern worldwide (Beyene 2016; Organization 2016; Song et al. 2016). Hence, the maximum residue limits of veterinary drugs in food products must be set (Sanders et al. 2016; Tang et al. 2017).

Veterinary drugs differ in type and chemical properties; thus, sample pretreatment and analytical techniques also differ (Moretti et al. 2016; Mainero et al. 2017). At present, analytical techniques used for veterinary drug residues in animal food sources are mainly based on HPLC (Kang et al. 2014; Samanidou et al. 2015), UPLC-MS/MS (Taylor et al. 2019; Zhao et al. 2018), UPLC-QE (Jia et al. 2017; Wang

Yingying Li

ying_sky@126.com

1 China Meat Research Center, 100068 Beijing, People's Republic of China et al. 2018a), UPLC-QTOF (Wang et al. 2018b; Kang et al. 2017), and GC-MS (Xue et al. 2017; Lozano et al. 2019). HPLC is inappropriate for the simultaneous detection of multiple residues of animal origin, and it can only be used for qualitative discrimination (Anumol et al. 2017). GC-MS is unsuitable for the detection of volatile, highly polar, and pyrolytic compounds. The most commonly used methods for detecting multi-residues in animal food sources include UPLC-MS/MS, UPLC-QE, and UPLC-QTOF (Xu et al. 2018). Steven used UPLC-MS/MS to detect 62 veterinary drug residues in the bovine kidney, with a recovery rate of $70 \%$ and a repeatability of less than $25 \%$ (Steven et al. 2012). Of the residues tested, 50 drugs met the qualitative confirmation criteria. No quantitative analysis was conducted in this method. Xie used HPLC-MS/MS to determine 54 veterinary drug residues in pork, belonging to classes of sulfonamides and quinolones. The method quantification limits range from $0.3 \mathrm{~g} / \mathrm{kg}$ to $3.0 \mathrm{~g} / \mathrm{kg}$, and the recovery rates range from $20.9 \%$ to $121.0 \%$ (Xie et al. 2012).

UPLC-MS/MS has insufficient anti-interference ability in a complex matrix and is inaccurate for the qualitative analysis of multi-residues in animal food sources. Compared with UPLC-MS/MS, high-resolution MS has better qualitative 
ability and can effectively avoid false-negative and falsepositive results.

High-resolution MS can be coupled to UPLC-QTOF and UPLC Q-Exactive, both of which determine the precise mass number of target compounds but differ in detection principle. Wang used UPLC-QTOF to determine the residues of aminoglycosides, $\beta$-lactams, and other veterinary drugs in milk and honey samples. This method can be used for the quantitative analysis of 31 and 34 compounds in honey and milk samples and conductand qualitative analysis of 54 and 34 compounds in honey and milk samples, respectively (Wang et al. 2012). Wang screened and quantified 125 veterinary drug residues in milk by using UPLC-Exactive-Orbitrap, with limits of quantification (LOQ) as low as $1.0 \mu \mathrm{g} / \mathrm{kg}$ (Wang et al. 2015).

This study aims to use UPLC Orbitrap Q-Exactive $\mathrm{HF}-\mathrm{X}$ as a rapid and accurate qualitative and quantitative method for detecting veterinary drug residues belonging to 21 classes in foods, such as pork, poultry meat, and their innards with simple pretreatment. Qualitative and quantitative results proved to be accurate, and the method was applied to authentic samples for the rapid detection of veterinary drug residues.

\section{Methods and Materials}

HPLC-grade methanol, acetonitrile (ACN), formic acid (FA), acetic acid, and ammonium acetate were procured from Thermo Fisher (USA). All veterinary drugs were obtained from Sigma-Aldrich (Germany).

Standard substances $(10.0 \mathrm{mg})$ were weighed and placed in a $10 \mathrm{~mL}$ brown volumetric flask. They were dissolved, volume-stabilized with appropriate reagents, such as methanol and ACN $1.0 \mathrm{mg} / \mathrm{mL}$ standard reserve solutions, and then stored at $-18^{\circ} \mathrm{C}$. The standard reserve solution prepared with water was stored at $4^{\circ} \mathrm{C}$. Intermediate veterinary drug standard mix working solution was prepared as $10.0 \mu \mathrm{g} /$ $\mathrm{mL}$ in methanol from stock solutions.

ACN $(800 \mathrm{~mL})$, water $(198 \mathrm{~mL})$, and FA $(2 \mathrm{~mL})$ were mixed to form a solution of ACN/water/FA $(80+19.8+0.2$, $\mathrm{v} / \mathrm{v})$. Water/FA $(99.9+0.1, \mathrm{v} / \mathrm{v})$ was prepared by mixing 999 $\mathrm{mL}$ of water with $1 \mathrm{~mL}$ of FA.

The samples were collected from abattoirs, supermarkets, or farmers' markets. A $2.0 \mathrm{~g}$ sample was weighed into a 50 $\mathrm{mL}$ centrifuge tube. Then, $10 \mathrm{~mL}$ of ACN-FA solution was added into the sample, which was shaken on a mechanical shaker. After $30 \mathrm{~min}$, the sample mixture was centrifuged at $4000 \mathrm{r} / \mathrm{min}$ for $3 \mathrm{~min}$ in a centrifuge. The supernatant $(5 \mathrm{~mL})$ was transferred into a PRiME HLB SPE column. The effluent was collected and blow-dried under nitrogen. The extracts were diluted up to $0.5 \mathrm{~mL}$ with water/FA $(99.9+0.1$, v/v) and vortexed for $30 \mathrm{~s}$. Sample extracts were analyzed using a UPLC-Q-Exactive Orbitrap/MS system.

A Thermo Scientific Q-Exactive HF-X was used to detect veterinary drugs. Veterinary drug residue analysis was performed with a Thermo Scientific Vanquish UHPLC, and MS analyses were conducted using Thermo Scientific Q-Exactive HF coupled to a Nanospray Flex ion source. The extract ion chromatograms of 155 veterinary drugs were shown in supporting information (Fig. S1).

In the positive mode, the mobile phase A was $0.1 \% \mathrm{FA} /$ $\mathrm{H}_{2} \mathrm{O}$; in the negative mode, the mobile phase A was $5 \mathrm{mM}$ ammonium acetate and the mobile phase $\mathrm{B}$ was ACN. The gradient profile was as follows: $0 \mathrm{~min}$ : $98 \% \mathrm{~A}$; 0-20 $\mathrm{min}$ : 98\%-0\% A; 20-25 min: 0\% A; 25-28 min: $98 \%$ A. The flow rate was $0.5 \mathrm{~mL} / \mathrm{min}$, and the injection volume was $5 \mu \mathrm{L}$. The UPLC column was an Agilent Eclipse plus C18, $3.0 \times 150 \mathrm{~mm}$, $1.8 \mu \mathrm{m}$. The column oven temperature was set at $40^{\circ} \mathrm{C}$, and the auto-sampler temperature was set at $4^{\circ} \mathrm{C}$.

The QE MS parameters were as follows: ScanType, Full MS-dd-MS2; Polarity, Negative; Sheath gas flow rate, 40 L; Aux gas flow rate, $15 \mathrm{~L}$; Spray voltage, $3.5 \mathrm{KV}$; Capillary temp, $325^{\circ} \mathrm{C}$; Aux gas heater temp, $450^{\circ} \mathrm{C}$; Full MS Resolution, 60 000; dd-MS2 Resolution, 15 000. Quantitatively method, the exact ratio of the parent ion and the characteristic fragment ion of the target compound; qualitatively method, the peak area of parent ion.

The calibration curves were validated over the concentration range $1 \times$ LOD $-25 \times$ LOD for veterinary drug residues in animal source foods. The correlation coefficients of all the curves were greater than 0.99 , and the deviations of the backcalculated concentrations from their nominal values were within $\pm 15 \%$. Results were fitted to linear regression analysis using $1 / \mathrm{x}^{2}$ as the weighting factor.

With the peak area of the standard substance as the y-axis and the corresponding working liquid concentration $(\mathrm{ng} / \mathrm{mL})$ as the $\mathrm{x}$-axis, all veterinary drugs showed good linearity in their appropriate concentration range. Recovery is a function of additive concentration, and a signal-to-noise ratio of 3 is acceptable to estimate the detection limit of the method (see Table 1). In total, 155 types of veterinary drug standard solutions were added to the negative samples, and the LOD and LOQ were calculated by taking three times the standard deviation for LOD and 6 or 10 times the standard deviation for LOQ. Six samples were measured in parallel with each standard level. As shown in Table 1, the average recovery rate of 155 veterinary drugs was between $79.2 \%$ and $118.5 \%$, $\mathrm{RSD} \leq 15 \%$. 
Table 1 Linear range, detection limit, standard recovery, and relative standard deviation of 155 veterinary drug residues in pork samples $(\mathrm{n}=6)$

\begin{tabular}{|c|c|c|c|c|c|c|c|c|c|}
\hline \multirow[t]{2}{*}{ No } & \multirow[t]{2}{*}{ Compound } & \multirow[t]{2}{*}{$\begin{array}{l}\text { Linearity } \\
\text { range } \mathrm{ng} / \mathrm{mL}\end{array}$} & \multirow[t]{2}{*}{$\mathrm{LOD} \mu \mathrm{g} / \mathrm{kg}$} & \multicolumn{2}{|c|}{$\begin{array}{l}\text { Spiked } \\
3 \times \text { LOD }\end{array}$} & \multicolumn{2}{|c|}{$\begin{array}{l}\text { Spiked } \\
5 \times \text { LOD }\end{array}$} & \multicolumn{2}{|c|}{$\begin{array}{l}\text { Spiked } \\
10 \times \text { LOD }\end{array}$} \\
\hline & & & & $\mathrm{R} / \%$ & $\mathrm{RSD} / \%$ & $\mathrm{R} / \%$ & $\mathrm{RSD} / \%$ & $\mathrm{R} / \%$ & $\mathrm{RSD} / \%$ \\
\hline 1 & Sulfaphenazole & $10-250$ & 5.0 & 86.6 & 6.3 & 83.5 & 8.1 & 88.3 & 9.4 \\
\hline 2 & Sulfabenzamide & $10-250$ & 5.0 & 94.8 & 5.9 & 95.1 & 1.3 & 93.9 & 3.0 \\
\hline 3 & Sulfapyridine & $10-250$ & 5.0 & 104.4 & 3.4 & 93.7 & 3.6 & 90.4 & 6.7 \\
\hline 4 & Sulfacetamide & $10-250$ & 5.0 & 101.1 & 7.8 & 94.0 & 14.1 & 108.3 & 9.6 \\
\hline 5 & Sulfameter & $10-250$ & 5.0 & 100.5 & 4.7 & 96.1 & 2.8 & 83.6 & 2.7 \\
\hline 6 & Sulfamoxole & $10-250$ & 5.0 & 89.3 & 6.8 & 103.3 & 4.3 & 101.0 & 2.3 \\
\hline 7 & Sulfisoxazole & $10-250$ & 5.0 & 93.6 & 5.3 & 106.9 & 5.0 & 107.0 & 3.7 \\
\hline 8 & Sulfamethazine & $10-250$ & 5.0 & 86.2 & 8.2 & 100.8 & 5.6 & 80.8 & 10.3 \\
\hline 9 & Sulfisomidine & $10-250$ & 5.0 & 83.3 & 7.9 & 83.4 & 10.9 & 96.3 & 5.2 \\
\hline 10 & Sulfamethoxazole & $10-250$ & 5.0 & 92.3 & 9.9 & 95.0 & 11.5 & 102.6 & 13.6 \\
\hline 11 & Sulfamerazine & $10-250$ & 5.0 & 99.4 & 11.3 & 91.4 & 8.9 & 107.5 & 11.9 \\
\hline 12 & Sulfamethoxypyridazine & $10-250$ & 5.0 & 93.6 & 7.5 & 101.1 & 10.8 & 90.1 & 6.2 \\
\hline 13 & Sulfadimethoxypyrimidine & $10-250$ & 5.0 & 98.2 & 5.4 & 108.3 & 10.5 & 91.6 & 8.9 \\
\hline 14 & Sulfamonnomethoxine & $10-250$ & 5.0 & 91.8 & 3.8 & 90.4 & 7.9 & 92.4 & 9.3 \\
\hline 15 & Sulfaquinoxaline & $10-250$ & 5.0 & 86.2 & 6.7 & 80.8 & 12.8 & 84.1 & 12.0 \\
\hline 16 & Sulfadimoxine & $10-250$ & 5.0 & 89.0 & 9.1 & 98.9 & 7.3 & 98.5 & 5.7 \\
\hline 17 & Sulfaclozine & $10-250$ & 5.0 & 118.5 & 7.4 & 111.1 & 11.4 & 115.3 & 14.3 \\
\hline 18 & Sulfachloropyridazine & $10-250$ & 5.0 & 89.7 & 2.5 & 95.6 & 3.7 & 87.1 & 4.5 \\
\hline 19 & Sulfaguanidine & $10-250$ & 5.0 & 109.1 & 6.7 & 106.7 & 13.4 & 103.0 & 7.0 \\
\hline 20 & Sulfadiazine & $10-250$ & 5.0 & 95.7 & 5.8 & 93.0 & 9.2 & 90.5 & 0.6 \\
\hline 21 & Sulfathiazole & $10-250$ & 5.0 & 106.4 & 4.4 & 105.9 & 0.8 & 96.9 & 5.5 \\
\hline 22 & Sulfamethizole & $10-250$ & 5.0 & 98.5 & 7.1 & 105.9 & 8.9 & 107.0 & 9.9 \\
\hline 23 & Orbifloxacin & $6-150$ & 3.0 & 114.0 & 8.3 & 115.9 & 5.8 & 100.5 & 13.8 \\
\hline 24 & Danofloxacin & $6-150$ & 3.0 & 111.9 & 12.6 & 99.0 & 9.2 & 93.0 & 14.2 \\
\hline 25 & Enrofloxacin & $10-250$ & 5.0 & 107.1 & 10.5 & 93.5 & 9.0 & 110.5 & 4.9 \\
\hline 26 & Flumequine & $6-150$ & 3.0 & 111.3 & 4.4 & 98.3 & 4.3 & 108.6 & 5.4 \\
\hline 27 & Fleroxacin & $6-150$ & 3.0 & 88.7 & 7.1 & 108.1 & 3.8 & 90.5 & 2.5 \\
\hline 28 & Ciprofloxacin & $6-150$ & 3.0 & 88.0 & 1.5 & 99.9 & 2.8 & 101.5 & 3.4 \\
\hline 29 & Lomefloxacin & $6-150$ & 3.0 & 103.1 & 12.0 & 90.8 & 9.0 & 91.2 & 5.4 \\
\hline 30 & Nalidixic acid & $6-150$ & 3.0 & 88.3 & 4.8 & 91.2 & 8.7 & 90.5 & 2.4 \\
\hline 31 & Norfloxacin & $6-150$ & 3.0 & 101.0 & 12.9 & 113.1 & 6.7 & 104.0 & 6.9 \\
\hline 32 & Pefloxacin & $6-150$ & 3.0 & 103.8 & 3.4 & 97.5 & 5.2 & 99.1 & 7.9 \\
\hline 33 & Sarafloxacin & $6-150$ & 3.0 & 86.4 & 12.2 & 85.6 & 14.6 & 82.5 & 7.9 \\
\hline 34 & Difloxacin & $6-150$ & 3.0 & 112.4 & 8.3 & 107.4 & 11.5 & 102.7 & 12.7 \\
\hline 35 & Sparfloxacin & $6-150$ & 3.0 & 80.5 & 6.5 & 92.4 & 7.0 & 86.9 & 4.7 \\
\hline 36 & Enoxacin & $10-250$ & 5.0 & 101.0 & 2.4 & 107.9 & 7.3 & 108.1 & 5.1 \\
\hline 37 & Ofloxacin & $6-150$ & 3.0 & 98.4 & 12.2 & 90.8 & 6.1 & 86.1 & 10.9 \\
\hline 38 & Clenbuterol & $1-25$ & 0.5 & 92.7 & 11.0 & 85.0 & 7.3 & 104.2 & 12.0 \\
\hline 39 & Ractopamine & $1-25$ & 0.5 & 88.7 & 6.5 & 86.2 & 4.4 & 85.5 & 7.3 \\
\hline 40 & Clorprenaline & $1-25$ & 0.5 & 101.8 & 11.9 & 106.2 & 12.5 & 108.1 & 10.5 \\
\hline 41 & Penbutolol & $1-25$ & 0.5 & 80.3 & 3.1 & 79.7 & 7.5 & 85.0 & 6.5 \\
\hline 42 & Metaproterenol & $1-25$ & 0.5 & 106.5 & 12.3 & 107.1 & 10.2 & 98.6 & 10.9 \\
\hline 43 & Formoterol & $1-25$ & 0.5 & 101.3 & 6.1 & 104.2 & 4.6 & 86.8 & 15.2 \\
\hline 44 & Fenoterol & $1-25$ & 0.5 & 93.3 & 8.6 & 93.8 & 11.1 & 91.7 & 9.5 \\
\hline 45 & Cimbuterol & $1-25$ & 0.5 & 101.7 & 8.0 & 95.9 & 14.3 & 97.8 & 4.8 \\
\hline 46 & Bambuterol & $1-25$ & 0.5 & 87.4 & 4.1 & 94.6 & 8.7 & 109.0 & 8.7 \\
\hline 47 & Phenylethanolamine A & $1-25$ & 0.5 & 84.6 & 9.2 & 104.0 & 9.3 & 101.4 & 7.2 \\
\hline
\end{tabular}


Table 1 (continued)

\begin{tabular}{|c|c|c|c|c|c|c|c|c|c|}
\hline \multirow[t]{2}{*}{ No } & \multirow[t]{2}{*}{ Compound } & \multirow[t]{2}{*}{$\begin{array}{l}\text { Linearity } \\
\text { range } \mathrm{ng} / \mathrm{mL}\end{array}$} & \multirow[t]{2}{*}{$\mathrm{LOD} \mu \mathrm{g} / \mathrm{kg}$} & \multicolumn{2}{|c|}{$\begin{array}{l}\text { Spiked } \\
3 \times \text { LOD }\end{array}$} & \multicolumn{2}{|c|}{$\begin{array}{l}\text { Spiked } \\
5 \times \text { LOD }\end{array}$} & \multicolumn{2}{|c|}{$\begin{array}{l}\text { Spiked } \\
10 \times \text { LOD }\end{array}$} \\
\hline & & & & $\mathrm{R} / \%$ & $\mathrm{RSD} / \%$ & $\mathrm{R} / \%$ & $\mathrm{RSD} / \%$ & $\mathrm{R} / \%$ & $\mathrm{RSD} / \%$ \\
\hline 48 & Thiabendazole & $1-25$ & 0.5 & 86.5 & 6.7 & 110.6 & 12.9 & 103.8 & 6.4 \\
\hline 49 & Salbutamol & $1-25$ & 0.5 & 108.4 & 9.6 & 93.1 & 11.9 & 101.3 & 12.7 \\
\hline 50 & Terbutaline & $2-50$ & 1.0 & 107.6 & 10.7 & 96.8 & 8.5 & 93.1 & 6.4 \\
\hline 51 & Tulobuterol & $1-25$ & 0.5 & 114.4 & 1.8 & 102.8 & 4.4 & 118.3 & 3.8 \\
\hline 52 & Cimaterol & $1-25$ & 0.5 & 104.6 & 9.2 & 111.7 & 6.2 & 95.8 & 6.4 \\
\hline 53 & Florfenicol & $10-250$ & 5.0 & 105.3 & 14.9 & 103.6 & 7.3 & 116.2 & 11.5 \\
\hline 54 & Chlortetracycline & $10-250$ & 5.0 & 98.6 & 11.4 & 104.0 & 4.5 & 100.7 & 8.7 \\
\hline 55 & Doxycycline & $10-250$ & 5.0 & 99.5 & 9.9 & 95.5 & 10.1 & 99.0 & 11.4 \\
\hline 56 & Tetracycline & $10-250$ & 5.0 & 93.2 & 4.9 & 105.0 & 7.5 & 103.0 & 13.0 \\
\hline 57 & Oxytetracycline & $10-250$ & 5.0 & 102.8 & 8.4 & 94.3 & 10.8 & 98.1 & 10.9 \\
\hline 58 & Erythromycin & $2-50$ & 1.0 & 80.1 & 3.0 & 86.7 & 6.6 & 79.9 & 6.4 \\
\hline 59 & Kitasamycin & $2-50$ & 1.0 & 91.1 & 12.9 & 84.2 & 14.6 & 95.0 & 5.5 \\
\hline 60 & Lincomycin & $2-50$ & 1.0 & 105.6 & 3.8 & 111.7 & 12.1 & 102.5 & 10.4 \\
\hline 61 & Tylosin & $2-50$ & 1.0 & 98.7 & 9.7 & 112.1 & 14.0 & 109.7 & 8.6 \\
\hline 62 & Tilmicosin & $2-50$ & 1.0 & 107.3 & 8.4 & 116.7 & 8.4 & 118.2 & 9.3 \\
\hline 63 & $\begin{array}{l}\text { Tylosin 3-acetate 4B-(3-methylbutanoate) } \\
\text { (2R,3R)-2,3-dihydroxybutanedioate }\end{array}$ & $2-50$ & 1.0 & 117.0 & 7.6 & 118.5 & 11.1 & 114.2 & 9.5 \\
\hline 64 & Oleandomycin & $2-50$ & 1.0 & 107.3 & 4.6 & 106.8 & 6.6 & 102.6 & 3.7 \\
\hline 65 & Ronidazole & $2-50$ & 1.0 & 99.7 & 3.6 & 92.7 & 5.1 & 101.8 & 3.4 \\
\hline 66 & Dimetridazole & $2-50$ & 1.0 & 111.3 & 8.4 & 104.9 & 7.8 & 108.8 & 5.3 \\
\hline 67 & Metronidazole-hydroxy & $2-50$ & 1.0 & 96.0 & 14.3 & 111.9 & 8.3 & 112.5 & 10.2 \\
\hline 68 & Dimetridazolr-hydroxy & $2-50$ & 1.0 & 97.2 & 11.6 & 105.7 & 9.5 & 103.5 & 11.8 \\
\hline 69 & Metronidazole & $2-50$ & 1.0 & 92.9 & 9.4 & 97.3 & 5.2 & 99.5 & 13.0 \\
\hline 70 & Oxfendazole & $10-250$ & 5.0 & 106.7 & 11.8 & 104.5 & 4.8 & 106.6 & 6.7 \\
\hline 71 & Febantel & $10-250$ & 5.0 & 83.2 & 9.6 & 79.6 & 14.7 & 86.0 & 13.3 \\
\hline 72 & Fenbendazole & $20-500$ & 10.0 & 101.7 & 7.6 & 108.9 & 11.1 & 106.9 & 10.5 \\
\hline 73 & Ampicillin & $10-250$ & 5.0 & 112.0 & 8.0 & 111.4 & 6.5 & 103.6 & 6.1 \\
\hline 74 & Oxacillin & $10-250$ & 5.0 & 96.2 & 10.9 & 90.5 & 11.2 & 97.8 & 7.2 \\
\hline 75 & cloxacillin & $10-250$ & 5.0 & 112.1 & 8.5 & 106.1 & 6.4 & 117.2 & 4.6 \\
\hline 76 & Dicloxacillin & $20-500$ & 10.0 & 97.2 & 12.8 & 93.2 & 10.7 & 92.0 & 7.9 \\
\hline 77 & Penicillin G & $2-50$ & 1.0 & 92.8 & 5.3 & 93.3 & 6.6 & 91.3 & 5.7 \\
\hline 78 & Cephapirin & $10-250$ & 5.0 & 100.0 & 10.7 & 110.6 & 9.2 & 107.7 & 7.9 \\
\hline 79 & Cefpirome & $2-50$ & 1.0 & 97.2 & 8.7 & 87.1 & 14.0 & 91.1 & 10.3 \\
\hline 80 & Ceftiofur & $20-500$ & 10.0 & 90.9 & 6.7 & 96.3 & 8.6 & 100.3 & 5.4 \\
\hline 81 & Cephalexin & $4-100$ & 2.0 & 101.6 & 13.1 & 103.0 & 12.7 & 107.9 & 8.8 \\
\hline 82 & 19-Nortestosterone & $2-50$ & 1.0 & 100.0 & 5.4 & 95.6 & 10.9 & 106.0 & 7.6 \\
\hline 83 & Medroxyprogesterone Acetate & $2-50$ & 1.0 & 79.9 & 10.6 & 82.7 & 12.5 & 85.9 & 8.4 \\
\hline 84 & Testosterone & $2-50$ & 1.0 & 94.8 & 6.1 & 90.3 & 10.0 & 87.7 & 4.5 \\
\hline 85 & 17-Methyltestosterone & $2-50$ & 1.0 & 99.7 & 3.7 & 104.9 & 6.1 & 89.7 & 5.8 \\
\hline 86 & Chlorpromazine & $1-25$ & 0.5 & 84.7 & 3.2 & 80.7 & 5.0 & 87.8 & 4.7 \\
\hline 87 & Azaperone & $0.4-10$ & 0.2 & 91.7 & 9.3 & 94.9 & 14.3 & 86.4 & 12.1 \\
\hline 88 & Promethazine & $2-50$ & 1.0 & 89.8 & 6.5 & 89.1 & 6.6 & 92.4 & 4.5 \\
\hline 89 & Acetopromaizine & $1-25$ & 0.5 & 82.8 & 5.5 & 80.3 & 11.2 & 79.7 & 10.1 \\
\hline 90 & Diazepam & $10-250$ & 5.0 & 90.9 & 11.1 & 106.7 & 10.9 & 116.1 & 14.3 \\
\hline 91 & Doramectin & $20-500$ & 10.0 & 111.4 & 10.4 & 96.8 & 9.9 & 107.7 & 11.0 \\
\hline 92 & Ivermectin & $20-500$ & 10.0 & 90.6 & 7.4 & 106.1 & 11.9 & 107.6 & 8.2 \\
\hline 93 & Maduramicin ammonium & $20-500$ & 10.0 & 104.5 & 8.1 & 113.3 & 10.1 & 106.2 & 6.8 \\
\hline 94 & Salinomycin & $20-500$ & 10.0 & 108.8 & 9.3 & 100.7 & 4.5 & 91.8 & 12.5 \\
\hline
\end{tabular}


Table 1 (continued)

\begin{tabular}{|c|c|c|c|c|c|c|c|c|c|}
\hline \multirow[t]{2}{*}{ No } & \multirow[t]{2}{*}{ Compound } & \multirow[t]{2}{*}{$\begin{array}{l}\text { Linearity } \\
\text { range } \mathrm{ng} / \mathrm{mL}\end{array}$} & \multirow[t]{2}{*}{$\mathrm{LOD} \mu \mathrm{g} / \mathrm{kg}$} & \multicolumn{2}{|c|}{$\begin{array}{l}\text { Spiked } \\
3 \times \text { LOD }\end{array}$} & \multicolumn{2}{|c|}{$\begin{array}{l}\text { Spiked } \\
5 \times \text { LOD }\end{array}$} & \multicolumn{2}{|c|}{$\begin{array}{l}\text { Spiked } \\
10 \times \text { LOD }\end{array}$} \\
\hline & & & & $\mathrm{R} / \%$ & RSD/\% & $\mathrm{R} / \%$ & $\mathrm{RSD} / \%$ & $\mathrm{R} / \%$ & $\mathrm{RSD} / \%$ \\
\hline 95 & Monensin & $20-500$ & 10.0 & 89.3 & 10.1 & 85.5 & 9.0 & 107.4 & 12.1 \\
\hline 96 & 2-Quinoxalinecarbox & $2-50$ & 1.0 & 89.7 & 5.6 & 88.0 & 3.6 & 80.0 & 9.1 \\
\hline 97 & Carbadox & $1-25$ & 0.5 & 83.9 & 14.8 & 80.1 & 8.2 & 80.3 & 11.9 \\
\hline 98 & Olaquindox & $10-250$ & 5.0 & 109.3 & 12.3 & 98.4 & 14.2 & 101.4 & 11.5 \\
\hline 99 & Desoxycarbadox & $1-25$ & 0.5 & 86.3 & 10.1 & 95.1 & 11.5 & 107.8 & 12.2 \\
\hline 100 & levamisole & $10-250$ & 5.0 & 85.8 & 9.8 & 83.2 & 7.3 & 86.4 & 12.0 \\
\hline 101 & Carbofuran & $2-50$ & 1.0 & 97.9 & 9.4 & 96.8 & 12.9 & 86.9 & 9.5 \\
\hline 102 & Coumaphos & $20-500$ & 10.0 & 80.4 & 4.6 & 79.5 & 6.6 & 83.5 & 8.4 \\
\hline 103 & Fenthion-sulfone & $20-500$ & 10.0 & 90.2 & 4.0 & 97.3 & 7.7 & 90.5 & 9.1 \\
\hline 104 & Fenthion-sulfoxide & $2-50$ & 1.0 & 93.4 & 14.7 & 88.8 & 10.3 & 91.2 & 12.1 \\
\hline 105 & Malathion & $2-50$ & 1.0 & 81.2 & 10.9 & 82.9 & 10.8 & 81.6 & 14.4 \\
\hline 106 & Phoxim & $2-50$ & 1.0 & 82.8 & 5.0 & 79.2 & 5.3 & 80.6 & 11.2 \\
\hline 107 & Dipterex & $2-50$ & 1.0 & 103.9 & 9.5 & 107.2 & 4.0 & 101.6 & 7.6 \\
\hline 108 & Trimethoprim & $2-50$ & 1.0 & 118.0 & 7.4 & 113.1 & 12.7 & 116.8 & 11.3 \\
\hline 109 & Atropine & $1-25$ & 0.5 & 112.4 & 3.4 & 110.4 & 4.0 & 112.1 & 3.1 \\
\hline 110 & Procaine & $2-50$ & 1.0 & 88.1 & 2.2 & 89.3 & 8.2 & 88.9 & 4.0 \\
\hline 111 & Lignocaine & $1-25$ & 0.5 & 96.4 & 11.7 & 91.8 & 7.9 & 109.8 & 12.1 \\
\hline 112 & Scopolamine & $1-25$ & 0.5 & 82.1 & 10.4 & 91.7 & 5.7 & 87.5 & 6.7 \\
\hline 113 & Anisodamine & $1-25$ & 0.5 & 99.0 & 5.5 & 89.8 & 1.5 & 106.8 & 6.7 \\
\hline 114 & Sulfanilamide & $20-500$ & 10.0 & 112.4 & 11.0 & 111.7 & 10.6 & 106.2 & 7.2 \\
\hline 115 & Mabuterol(Ambuterol) & $1-25$ & 0.5 & 90.2 & 6.3 & 97.7 & 12.3 & 92.5 & 10.6 \\
\hline 116 & Cefazolin & $2-50$ & 1.0 & 96.5 & 4.7 & 86.7 & 9.9 & 89.3 & 3.4 \\
\hline 117 & Amantadine & $2-50$ & 1.0 & 110.8 & 10.4 & 118.1 & 7.5 & 100.6 & 14.8 \\
\hline 118 & Rimantadine & $2-50$ & 1.0 & 107.4 & 11.7 & 100.6 & 13.7 & 97.1 & 8.2 \\
\hline 119 & Ribavirin & $4-100$ & 2.0 & 82.7 & 4.9 & 84.0 & 5.7 & 79.8 & 2.9 \\
\hline 120 & Oseltamivir & $2-50$ & 1.0 & 113.4 & 8.0 & 100.5 & 6.7 & 115.5 & 3.3 \\
\hline 121 & 4-Epi-Oxytetracycline & $10-250$ & 5.0 & 95.4 & 6.8 & 97.8 & 7.9 & 108.9 & 2.5 \\
\hline 122 & 4-Epi-Chlortetracycline & $10-250$ & 5.0 & 105.1 & 4.6 & 117.2 & 7.5 & 104.2 & 5.3 \\
\hline 123 & 4-Epi-Demeclocycline & $10-250$ & 5.0 & 101.6 & 2.8 & 114.3 & 4.6 & 103.9 & 7.6 \\
\hline 124 & Nequinate & $20-500$ & 10.0 & 80.1 & 10.0 & 85.7 & 11.0 & 81.9 & 4.6 \\
\hline 125 & Clopidol & $10-250$ & 5.0 & 110.7 & 13.3 & 109.4 & 14.9 & 105.6 & 9.4 \\
\hline 126 & Amprolium & $10-250$ & 5.0 & 101.4 & 12.6 & 101.6 & 7.4 & 114.0 & 9.0 \\
\hline 127 & Halofuginone hydrobromide & $10-250$ & 5.0 & 95.5 & 2.5 & 85.6 & 1.5 & 96.2 & 1.1 \\
\hline 128 & Narasin & $20-500$ & 10.0 & 81.1 & 4.3 & 79.6 & 11.6 & 82.7 & 7.8 \\
\hline 129 & Albendazole-2-aminosulfone & $4-100$ & 2.0 & 105.1 & 9.1 & 108.9 & 4.0 & 105.3 & 8.2 \\
\hline 130 & Albendazole sulfone & $2-50$ & 1.0 & 92.3 & 8.2 & 100.2 & 5.1 & 105.8 & 10.2 \\
\hline 131 & Albendazole sulfoxide & $10-250$ & 5.0 & 97.7 & 5.0 & 114.8 & 5.0 & 115.8 & 2.7 \\
\hline 132 & Albendazole & $20-500$ & 10.0 & 85.4 & 1.1 & 81.8 & 5.2 & 80.8 & 4.9 \\
\hline 133 & Diclazuril & $20-500$ & 10.0 & 89.3 & 2.5 & 100.4 & 4.1 & 93.4 & 6.3 \\
\hline 134 & Chloramphenicol & $0.2-5$ & 0.1 & 102.6 & 13.1 & 110.8 & 14.1 & 99.1 & 5.5 \\
\hline 135 & Beclomethasone & $2-50$ & 1.0 & 101.1 & 6.7 & 100.1 & 4.8 & 112.9 & 7.8 \\
\hline 136 & Cortisone acetate & $1-25$ & 0.5 & 114.9 & 6.7 & 94.3 & 5.5 & 91.9 & 2.8 \\
\hline 137 & Dexamethasone & $1-25$ & 0.5 & 91.9 & 14.1 & 97.6 & 7.6 & 96.9 & 7.1 \\
\hline 138 & Methylprednisolone & $1-25$ & 0.5 & 101.7 & 6.6 & 97.7 & 8.1 & 91.8 & 10.1 \\
\hline 139 & Cortisone & $1-25$ & 0.5 & 117.7 & 4.7 & 111.6 & 10.0 & 105.8 & 15.2 \\
\hline 140 & Meprednisone & $10-250$ & 5.0 & 112.8 & 2.0 & 114.9 & 9.1 & 95.7 & 9.2 \\
\hline 141 & Hydrocortisone & $2-50$ & 1.0 & 95.4 & 3.1 & 92.4 & 8.1 & 105.4 & 11.0 \\
\hline
\end{tabular}


Table 1 (continued)

\begin{tabular}{|c|c|c|c|c|c|c|c|c|c|}
\hline \multirow[t]{2}{*}{ No } & \multirow[t]{2}{*}{ Compound } & \multirow[t]{2}{*}{$\begin{array}{l}\text { Linearity } \\
\text { range } \mathrm{ng} / \mathrm{mL}\end{array}$} & \multirow[t]{2}{*}{$\mathrm{LOD} \mu \mathrm{g} / \mathrm{kg}$} & \multicolumn{2}{|c|}{$\begin{array}{l}\text { Spiked } \\
3 \times \text { LOD }\end{array}$} & \multicolumn{2}{|c|}{$\begin{array}{l}\text { Spiked } \\
5 \times \text { LOD }\end{array}$} & \multicolumn{2}{|c|}{$\begin{array}{l}\text { Spiked } \\
10 \times \text { LOD }\end{array}$} \\
\hline & & & & $\mathrm{R} / \%$ & $\mathrm{RSD} / \%$ & $\mathrm{R} / \%$ & $\mathrm{RSD} / \%$ & $\mathrm{R} / \%$ & $\mathrm{RSD} / \%$ \\
\hline 142 & Fludrocortisone acetate & $10-250$ & 5.0 & 96.3 & 10.0 & 93.8 & 13.3 & 104.6 & 2.5 \\
\hline 143 & Betamethasone & $1-25$ & 0.5 & 111.5 & 2.4 & 96.7 & 8.9 & 105.4 & 6.5 \\
\hline 144 & Diethylstilbestrol & $20-500$ & 10.0 & 94.4 & 4.3 & 95.1 & 7.7 & 89.3 & 7.4 \\
\hline 145 & Estradiol & $20-500$ & 10.0 & 95.4 & 6.7 & 88.6 & 4.5 & 90.7 & 13.7 \\
\hline 146 & Hexestrol & $20-500$ & 10.0 & 80.9 & 12.9 & 81.8 & 9.7 & 86.8 & 13.3 \\
\hline 147 & Lasalocid & $20-500$ & 10.0 & 85.6 & 3.1 & 86.4 & 4.5 & 83.5 & 4.3 \\
\hline 148 & Fipronil & $2-50$ & 1.0 & 97.0 & 4.2 & 93.8 & 10.3 & 100.5 & 8.0 \\
\hline 149 & Clazuril & $20-500$ & 10.0 & 96.7 & 9.9 & 93.8 & 13.1 & 102.8 & 11.8 \\
\hline 150 & Nicarbazin & $2-50$ & 1.0 & 88.0 & 2.0 & 82.9 & 4.2 & 80.9 & 2.2 \\
\hline 151 & Fipronil sulfone & $4-100$ & 2.0 & 92.5 & 6.8 & 97.9 & 3.9 & 83.0 & 7.3 \\
\hline 152 & Fipronil sulfide & $4-100$ & 2.0 & 111.3 & 4.0 & 104.7 & 8.1 & 115.9 & 13.0 \\
\hline 153 & Fipronil desulfinyl & $4-100$ & 2.0 & 110.5 & 9.5 & 108.3 & 11.0 & 98.1 & 12.1 \\
\hline 154 & Thiamphenicol & $2-50$ & 1.0 & 98.8 & 11.0 & 94.4 & 10.9 & 92.9 & 7.1 \\
\hline 155 & Abamectin & $20-500$ & 10.0 & 98.3 & 12.1 & 85.5 & 6.5 & 103.5 & 5.1 \\
\hline
\end{tabular}

\section{Results and Discussion}

The 155 veterinary drugs selected in this study cover an extremely wide polarity range. Thus, chromatographic columns with good retention for compounds of different polarity degrees must be selected. First, the separation effects of 155 compounds on three chromatographic columns, including a Waters Acquity UPLC BEH C18 column, a Thermo Hypersil GOLD column, and an Agilent ZORBAX Eclipse Plus C18 column, were compared. Results showed that the separation effects and peak shapes for the Agilent ZORBAX Eclipse Plus C18 column were superior to those of the two other chromatographic columns. Furthermore, the mobile phases $(0.1 \%$ FA aqueous solution and $5 \mathrm{mM}$ ammonium acetate) were optimized. Organic solvents investigated were $\mathrm{ACN}$ and methanol, as well as mixtures of ACN solution containing $0.1 \%$ FA and methanol containing $0.1 \%$ FA solution. In the positive mode, $0.1 \% \mathrm{FA}$ aqueous solution and ACN solution containing $0.1 \%$ FA performed best in separating the target compounds; in the negative mode, $5 \mathrm{mM}$ ammonium acetate and $\mathrm{ACN}$ performed best. The ion flow chromatogram of 155 target compounds is shown in Fig. 1.

Adding an appropriate amount of salt in the mobile phases can help improve the peak shape of the mass spectrum and improve the ionization efficiency of the substance to be measured. However, the ionization efficiency reduced when the concentration of salt was too high, thus resulting in the mass spectrum response of the substance to be measured. Ammonium acetate and $\mathrm{ACN}$ at $0,2,5$ and $10 \mathrm{mM}$ were selected as mobile phases, respectively, to investigate the chromatographic peak shape and mass spectral response intensity of each compound in the negative mode. As shown in Figs. 2 and 3, when the ammonium acetate concentration in the aqueous mobile phase was $5 \mathrm{mM}$, the responses of abamectin and hexestrol were significantly higher than those of pure water or other concentrations of ammonium acetate. Therefore, $5 \mathrm{mM}$ ammonium acetate and $\mathrm{ACN}$ were selected as the mobile phases in the negative mode in this experiment.

The target compounds were quantitatively and qualitatively analyzed by MS. Thus, isomers were required to achieve baseline separation on the chromatographic column. Four pairs of sulfa compounds are isomers of each other (Nos. 6 and 7, 8 and 9, 13 and 16, 17 and 18), and three compounds are isomers of each other (Nos. 5, 12, and 14). Baseline separation was achieved under selected chromatographic conditions, and retention times are shown in supporting information (Table S1).

Sulfameter, sulfamethoxypyridazine, and sulfamonomethoxine are isomers with identical plasmonucleic ratios, which must be separated by chromatographic retention time. In this study, the chromatographic conditions were optimized to achieve baseline separation. From the extracted ion chromatogram (Fig. 4), the three sulfamides were determined by peak comparison with reference standards.

The Q-Exactive Orbitrap/MS was operated in Full MS/ dd MS2 scanning mode in the positive and negative modes. Targeted identification was achieved by full-scan MS; if a veterinary drug was detected, the data-dependent MS2 (ddMS2) scan was triggered.

During the Full MS scan, the mass resolution was set at 60,000 FWHM, AGC target at 1.0E6, maximum IT 200 $\mathrm{ms}$, and scan range $\mathrm{m} / \mathrm{z} 135-1100$. If the targeted compound was detected within a 5 ppm mass error window 
Fig. 1 Total ion current chromatogram of 155 veterinary drugs

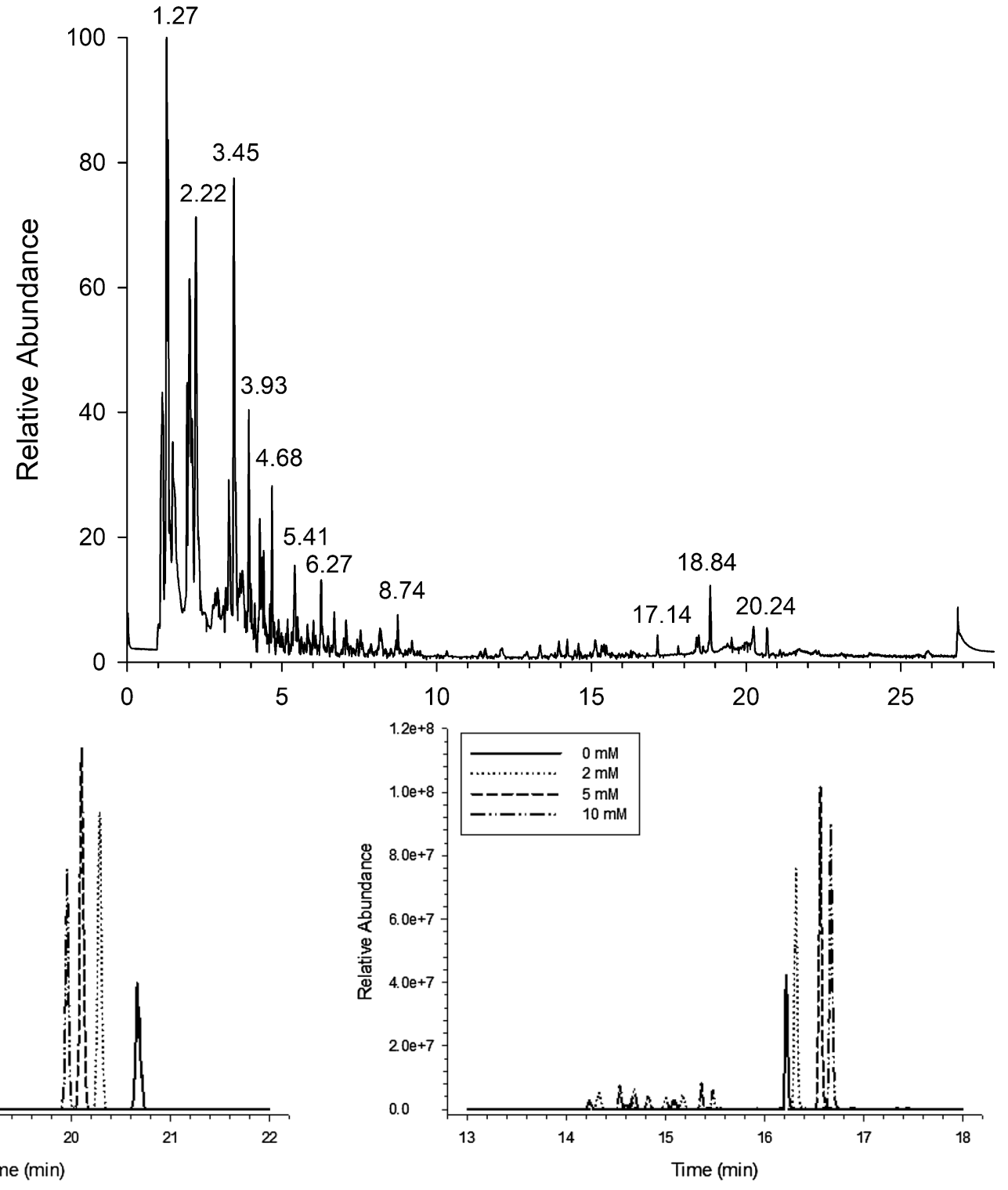

Fig. 3 Effect of ammonium acetate concentration in mobile phase on quality spectrum response of hexestrol

An appropriate extraction solvent needs to consider the properties of the solvent, veterinary drugs, and matrix. Organic solvents, such as ACN, ethyl acetate, ACN-aqueous solution, and $\mathrm{ACN}-0.2 \%$ formic water $(8+2)$ solution, were selected to extract different matrix samples. Figure 5 shows the extraction efficiencies and recovery rates of the 155 compounds in the different solvents. Results showed that $\mathrm{ACN}-0.2 \%$ formic water $(8+2)$ solution recovered more compounds in a more appropriate range $(80<\mathrm{R}<120)$ compared with the other solvents.

The fats and proteins in animal-derived food must be removed during purification because they increase the matrix effect, reduce the sensitivity of the method, pollute the mass spectrum, and shorten the service life of the 


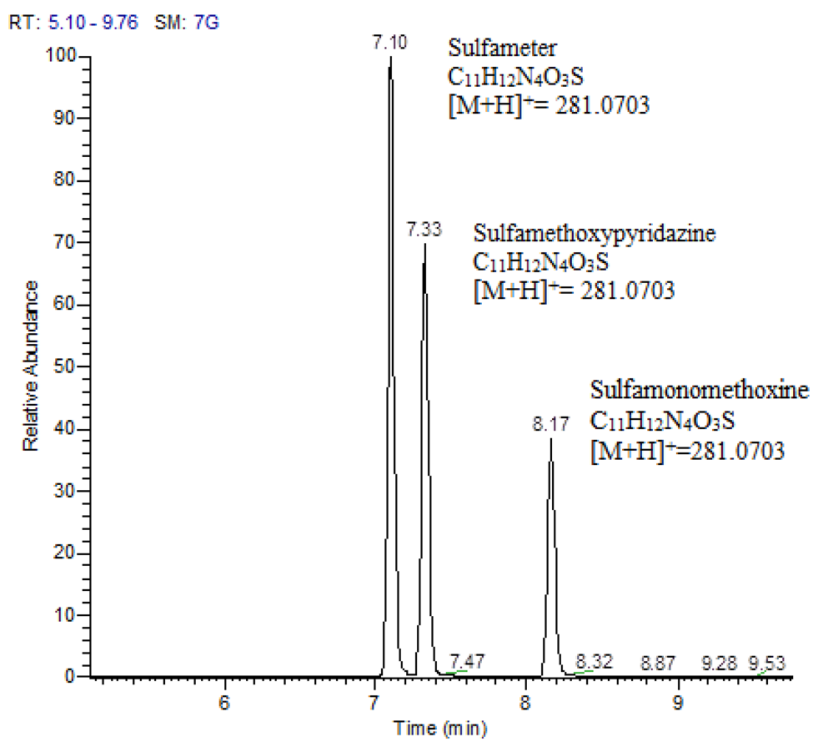

Fig. 4 Extracted ion chromatogram of the exact mass of 281.0703 (sulfameter, sulfamethoxypyridazine, or sulfamonomethoxine)

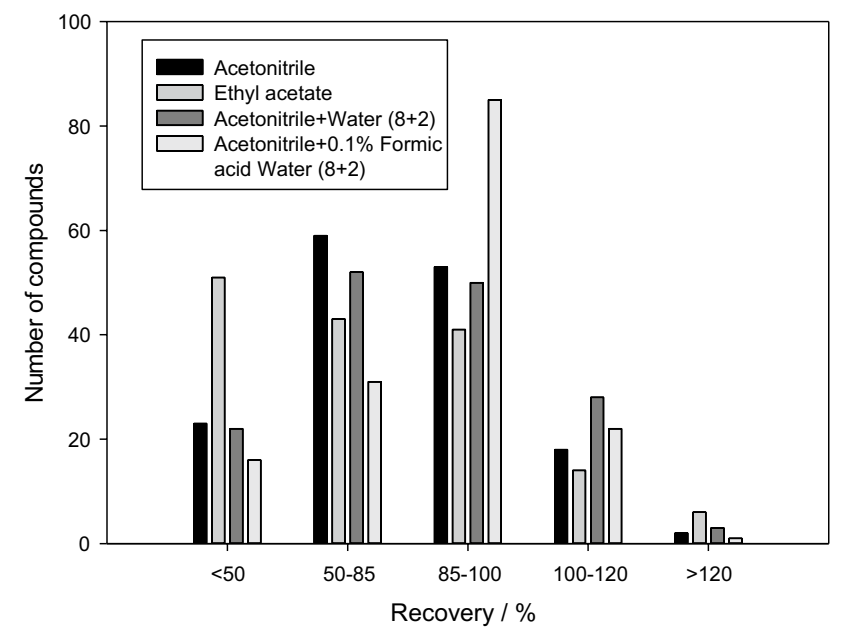

Fig. 5 Number of compounds extracted with different extraction solvents

instrument. Four purification methods, including QuEChERs purification, PRiME HLB SPE column, HyperSep Retain PEP SPE column, and Favex-AG column purification, were selected and evaluated, and the results are shown in Fig. 6. More compounds were recovered when the PRiME HLB SPE column was used for purification, where the recovery rate was within a reasonable range. Therefore, the PRiME HLB SPE column was selected as the purification method.

A calibration curve was prepared to compare the three methods, namely, using solvent standard solution, blank matrix standard solution, and add standard solution before purification, for the quantitative correction and calculation

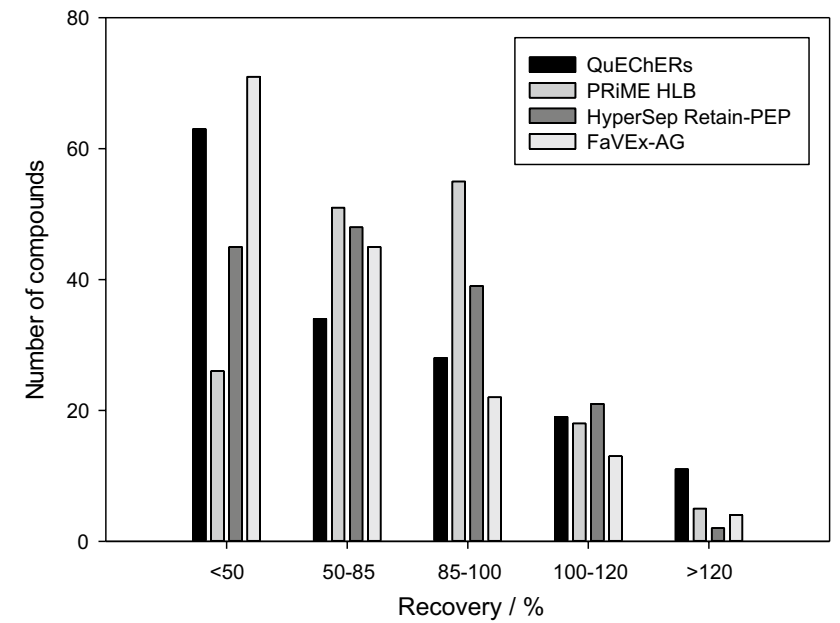

Fig. 6 Number of compounds extracted with the different clean-up approaches evaluated

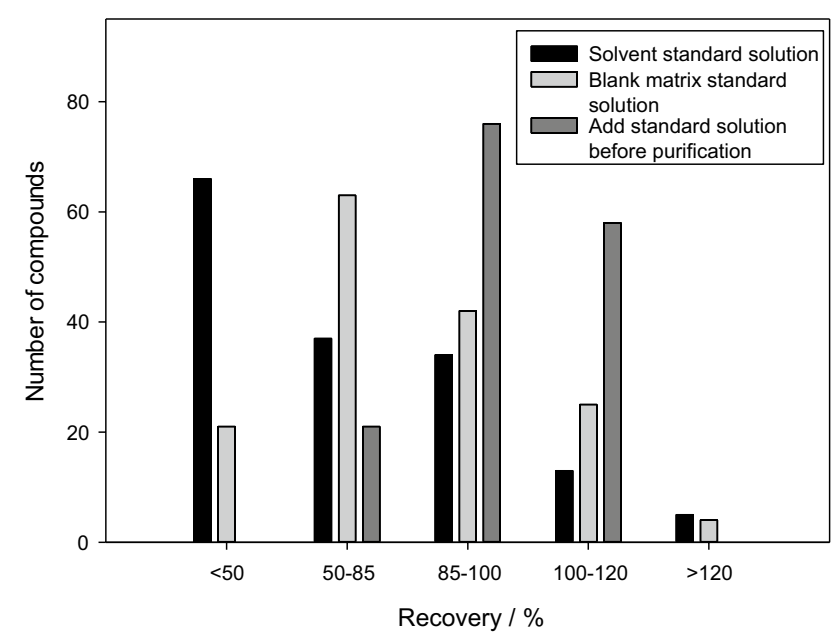

Fig. 7 Number of compounds extracted with the different standard curve preparation methods

of the recovery rates of the veterinary drugs. The results are shown in Fig. 7.

When the standard curve of the solvent standard solution method was used for quantitative detection, the recovery rates of most of the 155 veterinary drugs were low because the preparation method could not correct matrix effects and because of loss of veterinary drugs during the pretreatment. For the standard curve of the blank matrix standard solution method, the recovery rate was significantly higher than that of the solvent standard solution method because it could reduce the influence of matrix effects but could not eliminate the loss of most veterinary drugs during the pretreatment. It still could not meet the need of accurate quantification. In the present study, a standard solution was added before pretreatment for the preparation of the standard curve. This method 
can not only reduce matrix effects but also effectively reduce wastage of the target compounds during pretreatment.

The method was applied to 195 meat and viscera samples, in cloud pork, beef, mutton, chicken, pork liver, and chicken liver. The sample information is provided in supporting information (Table S2). The results are shown in Table 2. Fourteen classes of veterinary drugs, such as quinolones, agonists, tetracyclines, sulfamides, and glucocorticoids, were detected in the samples, among which hydrocortisone and cortisone were endogenous glucocorticoids. Meanwhile, 22 classes of veterinary drugs were detected in 70 samples, with a detection rate of $35.9 \%$. Aureomycin was detected in 31 samples, which was the most veterinary drug detected, with contents ranging from $5.03 \mu \mathrm{g} / \mathrm{kg}$ to $147 \mu \mathrm{g} / \mathrm{kg}$. Its metabolite chlorquatrimycin was detected in 21 samples, ranging from $5.70 \mu \mathrm{g} / \mathrm{kg}$ to $157 \mu \mathrm{g} / \mathrm{kg}$. Timicoxacin was detected in 13 samples, mainly concentrated in pig liver and chicken liver, ranging from $1.11 \mu \mathrm{g} / \mathrm{kg}$ to $179 \mu \mathrm{g} / \mathrm{kg}$. The detection rates of enoxacin and doxycycline were also relatively high.

Therefore, the detection rates of veterinary drug residues in animal source foods rapidly screened by highresolution MR are higher than those for other methods because of its higher sensitivity than HPLC-MS/MS and lower LOD than conventional detection standards. Moreover, it covered 155 types of veterinary drugs. Thus, such a detection range cannot be achieved by conventional detection methods. In addition, a large number of veterinary drugs were detected in 195 authentic samples, but the values of the results were less than the LOQ. These data may provide insights into breeding animals for human consumption.

Table 2 Veterinary drug detection in authentic samples

\begin{tabular}{|c|c|c|c|c|c|c|c|}
\hline $\begin{array}{l}\text { Animal spe- } \\
\text { cies (Number of } \\
\text { samples) }\end{array}$ & Analyte & $\begin{array}{l}\text { Number } \\
\text { of positive } \\
\text { samples }\end{array}$ & $\begin{array}{l}\text { Content range } \\
\mu \mathrm{g} / \mathrm{kg}\end{array}$ & $\begin{array}{l}\text { Animal spe- } \\
\text { cies (Number of } \\
\text { samples) }\end{array}$ & Analyte & $\begin{array}{l}\text { Number } \\
\text { of positive } \\
\text { samples }\end{array}$ & $\begin{array}{l}\text { Content range } \\
\mu \mathrm{g} / \mathrm{kg}\end{array}$ \\
\hline \multirow[t]{15}{*}{ Beef (40) } & Hydrocortisone & 34 & $1.36-45.4$ & \multirow[t]{8}{*}{ Pork (37) } & Hydrocortisone & 36 & $1.27-13.9$ \\
\hline & Cortisone & 6 & $1.07-3.28$ & & Cortisone & 5 & $1.19-4.13$ \\
\hline & Atropine & 3 & $1.82-2.45$ & & Chlortetracycline & 5 & $5.08-11.2$ \\
\hline & Chlortetracycline & 2 & $5.66-339$ & & $\begin{array}{l}\text { 4-Epi-Chlortetra- } \\
\text { cycline }\end{array}$ & 3 & $6.12-7.33$ \\
\hline & Trimethoprim & 2 & $1.12-5.08$ & & Tilmicosin & 4 & $1.11-11.5$ \\
\hline & Enrofloxacin & 2 & $9.07-13.8$ & & Atropine & 1 & 1.91 \\
\hline & Clenbuterol & 1 & 1.05 & & Levamisole & 1 & 5.90 \\
\hline & Florfenicol & 1 & 51.8 & & Thiabendazole & 1 & 0.571 \\
\hline & Ciprofloxacin & 1 & 4.18 & \multirow[t]{7}{*}{ Mutton (36) } & Hydrocortisone & 32 & $1.22-13.5$ \\
\hline & Thiabendazole & 1 & 0.536 & & Chlortetracycline & 10 & $5.03-103$ \\
\hline & Doxycycline & 1 & 171 & & $\begin{array}{l}\text { 4-Epi-Chlortetra- } \\
\text { cycline }\end{array}$ & 7 & $5.70-56.2$ \\
\hline & Tetracycline & 1 & 14.2 & & Cortisone & 3 & $1.58-2.13$ \\
\hline & $\begin{array}{l}\text { 4-Epi-Chlortetra- } \\
\text { cycline }\end{array}$ & 1 & 157 & & Enrofloxacin & 2 & $3.88-4.06$ \\
\hline & Sulfisomidine & 1 & 21.6 & & Doxycycline & 1 & 132 \\
\hline & Testosterone & 1 & 1.07 & & Trimethoprim & 1 & 1.79 \\
\hline \multirow[t]{10}{*}{ Pork liver (36) } & Chlortetracycline & 11 & $6.21-147$ & \multirow[t]{4}{*}{ Chicken (24) } & Thiabendazole & 2 & $0.635-1.14$ \\
\hline & $\begin{array}{l}\text { 4-Epi-Chlortetra- } \\
\text { cycline }\end{array}$ & 9 & $8.79-103$ & & Enrofloxacin & 1 & 4.27 \\
\hline & Enrofloxacin & 5 & $3.75-8.05$ & & Oxytetracycline & 1 & 11.4 \\
\hline & Tilmicosin & 5 & $2.49-177$ & & Diclazuril & 1 & 26.0 \\
\hline & Olaquindox & 4 & $1.65-19.0$ & \multirow[t]{6}{*}{ Chicken liver (22) } & Tilmicosin & 4 & $1.45-179$ \\
\hline & Doxycycline & 3 & $8.46-29.6$ & & Chlortetracycline & 3 & $10.4-31.7$ \\
\hline & Ofloxacin & 1 & 225 & & $\begin{array}{l}\text { 4-Epi-Chlortetra- } \\
\text { cycline }\end{array}$ & 3 & $6.77-23.2$ \\
\hline & Lincomycin & 1 & 4.03 & & Doxycycline & 2 & $11.7-18.1$ \\
\hline & Trimethoprim & 1 & 1.2 & & Amantadine & 1 & 29.7 \\
\hline & Lignocaine & 1 & 0.505 & & Diclazuril & 1 & 320 \\
\hline
\end{tabular}




\section{Conclusions}

UPLC-Q-Exactive Orbitrap/MS was used to establish a fast method for the qualitative and quantitative analysis of 155 veterinary drug residues of different classes in animal source foods. With this method, high-resolution MS can ensure the elimination of interference in complex matrix backgrounds and significantly improve the accuracy of qualitative and quantitative analyses, and data-dependent, full-scan, secondary-ion, MS further improves the accuracy of qualitative results. This method can increase the detection flux, reduce the detection cost, shorten the detection cycle, and provide technical support for the rapid response to food safety problems and the detection of potential food safety risks.

Supplementary Information The online version of this article (https:// doi.org/10.1007/s00128-021-03273-w) contains supplementary material, which is available to authorized users.

Acknowledgements This work was funded by the National Key Research and Development Program of China (2017YFC1601706), and the project was supported by Beijing Postdoctoral Research Foundation.

Open Access This article is licensed under a Creative Commons Attribution 4.0 International License, which permits use, sharing, adaptation, distribution and reproduction in any medium or format, as long as you give appropriate credit to the original author(s) and the source, provide a link to the Creative Commons licence, and indicate if changes were made. The images or other third party material in this article are included in the article's Creative Commons licence, unless indicated otherwise in a credit line to the material. If material is not included in the article's Creative Commons licence and your intended use is not permitted by statutory regulation or exceeds the permitted use, you will need to obtain permission directly from the copyright holder. To view a copy of this licence, visit http://creativecommons.org/licenses/by/4.0/.

\section{References}

Anumol T, Lehotay S, Stevens J et al (2017) Comparison of veterinary drug residue results in animal tissues by ultrahigh-performance liquid chromatography coupled to triple quadrupole or quadrupole-time-of-flight tandem mass spectrometry after different sample preparation methods, including use of a commercial lipid removal product. Anal Bioanal Chem 409:2639-2653. https://doi.org/10.1007/s00216-017-0208-y

Beyene T (2016) Veterinary drug residues in food-animal products: its risk factors and potential effects on public health. Journal of Veterinary ence Technology 7:1-7. https://doi.org/10.4172/ 2157-7579.1000285

Jia W, Chu X, Chang J et al (2017) High-throughput untargeted screening of veterinary drug residues and metabolites in tilapia using high resolution orbitrap mass spectrometry. Anal Chim Acta 957:29-39. https://doi.org/10.1016/j.aca.2016.12.038

Kang J, Park S, Park H et al (2017) Multiresidue screening of veterinary drugs in meat, milk, egg, and fish using liquid chromatography coupled with ion trap time-of-flight mass spectrometry.
Appl Biochem Biotechnol 182:635-652. https://doi.org/10. 1007/s12010-016-2350-y

Kang Y, Yu J, Huh Y et al (2014) Development of high performance liquid chromatography-ultraviolet detection method for screening mebendazole, clorsulon, diaveridine, and tolfenamic acid in animal-based food samples. Drug Testing Analysis 6:246-256. https://doi.org/10.1002/dta.1467

Knap P (2020) The scientific development that we need in the animal breeding industry. J Anim Breed Genet 137:343-344. https:// doi.org/10.1111/jbg.12485

Kpodékon T, Ogni C, Dassou H et al (2015) Dominant viral pathologies in the extensive and semi-intensive animal breeding and their treatment mode in ethno veterinary medicine in benin. Veterinary World 8:1424-1434. https://doi.org/10.14202/vetwo rld.2015.1424-1434

Laranjo M, Elias M, Fraqueza M (2017) The Use of Starter Cultures in Traditional Meat Products. J Food Qual 2017:1-18. https://doi. org/10.1155/2017/9546026

Liu Z, Anthony N, Chen H (2019) Food safety governance in China: From supervision to coregulation. Food Sci Nutr 7:4127-4139. https://doi.org/10.1002/fsn3.1281

Lozano A, Hernando M, Ucles S et al (2019) Identification and measurement of veterinary drug residues in beehive products. Food Chem 274:61-70. https://doi.org/10.1016/j.foodchem.2018.08. 055

Mainero R, Gentili A, Pérez F et al (2017) Veterinary drugs residues: a review of the latest analytical research on sample preparation and lc-ms based methods. Food Additives Contaminants Part A Chemistry Analysis Control Exposure Risk Assessment 34:766-784. https://doi.org/10.1080/19440049.2017.1298846

Moretti S, Dusi G, Giusepponi D et al (2016) Screening and confirmatory method for multiclass determination of 62 antibiotics in meat. J Chromatogr A 1429:175-188. https://doi.org/10.1016/j.chroma. 2015.12.021

Organization W (2016) Evaluation of certain veterinary drug residues in food eighty-first report of the joint fao/who expert committee on food additives. World Health Organization Technical Report 997:1-110. https://doi.org/10.1515/ci.1999.21.2.49b

Samanidou V, Christodoulou E, Papadoyannis I (2015) Validation of a novel hplc sorbent material for the determination of ten quinolones in human and veterinary pharmaceutical formulations. J Sep ence 28:2444-2453. https://doi.org/10.1002/jssc.200500262

Sanders P, Jérôme H, Laurentie M (2016) Tools to evaluate pharmacokinetics data for establishing maximum residue limits for approved veterinary drugs: examples from jecfa's work. Drug Testing Analysis 8:565-571. https://doi.org/10.1002/dta.2006

Song J, Park S, Choi J et al (2016) Development of analytical method and monitoring of veterinary drug residues in korean animal products. Korean J Food Sci Ani Res 36:319-325. https://doi.org/10. 5851/kosfa.2016.36.3.319

Steven J, Alan R, Lucía G et al (2012) Development and validation of a streamlined method designed to detect residues of 62 veterinary drugs in bovine kidney using ultra-high performance liquid chromatography-tandem mass spectrometry. Drug Testing Analysis 4:75-90. https://doi.org/10.1002/dta.1363

Tang X, Zheng X, Wang W et al (2017) Current situations and development on standards of veterinary drugs maximum residue limits in animal products. J Food Sci Technol 035:8-12. https://doi.org/10. 3969/j.issn.2095-6002.2017.04.002. (in Chinese)

Taylor M, Giela A, Sharp E et al (2019) A rapid multi-class, multiresidue uhplc-ms/ms method for the simultaneous determination of anticoagulant rodenticides, pesticides and veterinary medicines in wild animals, pets and livestock. Anal Methods 11:1087-1101. https://doi.org/10.1039/c8ay02367k

Trishna P, Tara M, Ronette G et al (2018) Drug residues in poultry meat: a literature review of commonly used veterinary 
antibacterials and anthelmintics used in poultry. J Vet Pharmacol Ther 41:761-789. https://doi.org/10.1111/jvp.12700

Wang J, Leung D (2012) The challenges of developing a generic extraction procedure to analyze multi-class veterinary drug residues in milk and honey using ultra-high pressure liquid chromatography quadrupole time-of-flight mass spectrometry. Drug Testing Analysis 4:103-111. https://doi.org/10.1002/dta.1355

Wang J, Leung D, Chow W et al (2015) The development and validation of a multi-class method for analysis of veterinary drug residues in milk using ultra-high performance liquid chromatography electrospray ionization quadrupole orbitrap mass spectrometry. J Agric Food Chem 63:9175-9187. https://doi.org/10.1021/acs. jafc.5b04096

Wang J, Leung D, Chow W et al (2018a) Target screening of 105 veterinary drug residues in milk using UHPLC/ESI Q-Orbitrap multiplexing data independent acquisition. Anal Bioanal Chem 410:5373-5389. https://doi.org/10.1007/s00216-017-0847-z

Wang Z, Li Y, Chang Q et al (2018b) A detection and confirmation strategy for screening of veterinary drugs in honey by liquid chromatography coupled quadrupole time-of-flight mass spectrometry. Anal Methods 10:59-68. https://doi.org/10.1039/C7AY02440A

Xie W, Han C, Hou J et al (2015) Simultaneous determination of multiveterinary drug residues in pork meat by liquid chromatography-tandem mass spectrometry combined with solid phase extraction. J Sep ence 35:3447-3454. https://doi.org/10. 1002/jssc.201200407

Xu Z, Li J, Chen A et al (2018) A new retrospective, multi-evidence veterinary drug screening method using drift tube ion mobility mass spectrometry. Rapid Commun Mass Spectrom 30:11411148. https://doi.org/10.1002/rcm.8154

Xue L, Cai Q, Zheng X et al (2017) Determination of 9 Hydroxy Veterinary Drug Residues in Fish by QuEChERS-GPC-GC/MS. Journal of Chinese Mass Spectrometry Society 06:655-663. https:// doi.org/10.7538/zpxb.2016.0148.(in Chinese)

Zhao L, Lucas D, Long D et al (2018) Multi-class multi-residue analysis of veterinary drugs in meat using enhanced matrix removal lipid cleanup and liquid chromatography-tandem mass spectrometry. J Chromatogr A 1549:14-24. https://doi.org/10.1016/j. chroma.2018.03.033

Publisher's Note Springer Nature remains neutral with regard to jurisdictional claims in published maps and institutional affiliations. 